\title{
Plasmonic light enhancement in the near-field of metallic nanospheroids for application in intermediate band solar cells
}

\author{
Manuel J. Mendes, ${ }^{a)}$ Antonío Luque, Ignacio Tobías, and Antonio Martí \\ Instituto de Energía Solar, Universidad Politécnica de Madrid, E28040 Madrid, Spain
}

\begin{abstract}
In order to enhance infrared light absorption in sub-bandgap transitions in an intermediate band solar cell, the scattered near-field potential from uncoated and coated metallic nanoparticles with a spheroidal shape is calculated with the electrostatic model. The absorption enhancement produced at the surface plasmon frequency of the nanoparticles can be of several orders of magnitude in some cases.
\end{abstract}

Conventional single-gap solar cells cannot exploit photon energies below semiconductor bandgap energies. A promising concept to overcome this limitation is the intermediate band solar cell (IBSC), ${ }^{1}$ which reaches a detailed balance efficiency limit of $63.2 \%$ compared to $40.7 \%$ for singlegap cells. The IBSC can generate photocurrent from subbandgap photons, without voltage degradation, due to the existence of an electronic band-the intermediate band (IB) — within the semiconductor bandgap.

The IB can be formed by the confined levels of a quantum dot (QD) array. Prototype IBSCs were fabricated with ten QD layers. ${ }^{2}$ However, the IB impact on the cell performance is still marginal, mainly due to the weak absorption coefficient associated to the QDs. One way of increasing this absorption is to grow more QD layers, but this introduces strain-induced dislocations that deteriorate the device performance. ${ }^{3}$ Procedures for reducing the strain are in development, ${ }^{4-6}$ but this is still a challenging problem. In this contribution, an alternative procedure is studied that exploits the high near-field that can appear in the vicinity of metal nanoparticles (MNPs) sustaining surface plasmons. The inclusion of these particles close to QDs can amplify their absorption, allowing the replacement of several QD layers by a single one with MNPs. These MNPs might also induce defects but it is possible that the reduction of layers has an overall positive effect.

When an electric plane-wave impinges on a particle much smaller than its wavelength the electric field induced inside the particle can be assumed uniform. ${ }^{7}$ This is the principle of electrostatic approximation (EA), valid only if the following conditions are simultaneously fulfilled:

$$
2 \pi r_{\mathrm{eq}} / \lambda \ll 1 \text { and }\left|N_{r}\right| 2 \pi r_{\mathrm{eq}} / \lambda \ll 1
$$

where $r_{\text {eq }}$ is a characteristic length of the particle, taken as the radius of the volume-equivalent sphere, ${ }^{8} \lambda$ is the wavelength in the medium, and $N_{r}$ the complex refractive index of the particle divided by that of the medium. In this paper, we consider $\mathrm{Ag} \mathrm{MNP}$ in a GaAs medium. $\mathrm{Ag}$ is one of the metals with strongest plasmonic scattering at solar frequencies ${ }^{9}$ and most IBSC have InAs QDs in a GaAs matrix. The refractive index of these materials is interpolated from experimental data. ${ }^{10}$ The values of $r_{\mathrm{eq}}$ that set conditions (1) equal to unity are given in Fig. 1(a). EA solutions can be consid-

\footnotetext{
${ }^{a)}$ Electronic mail: manuel.mendes@ies-def.upm.es.
}

ered rather accurate for particle $r_{\text {eq }}$ up the lowest of the two curves in the figure. ${ }^{8}$

In EA the scattered potential $\phi_{S}$ is obtained from Laplace equation $(\nabla \cdot \mathbf{E}=0)$ whose solution is exact for ellipsoids with semiaxes $a, b$, and $c$. Due to the unpolarized nature of sunlight, the incident field $\boldsymbol{E}_{0}$ can assume any orientation orthogonal to the propagation direction $\boldsymbol{K}_{0}$ [see Fig. $1(\mathrm{~b})]$.

For our scattering object to respond equally to any such polarizations, the MNP must be spheroidal (ellipsoid with $a=b)$ either oblate $(a=b>c)$ or prolate $(c>a=b)$ having the $c$ axis along $\boldsymbol{K}_{0}$.

Although solar cells may receive sunlight with oblique incidence, even oblique rays become almost normal when entering the semiconductor due to its high refractive index. Thus, the analysis presented here is approximately valid for this case.

The potential scattered by a spheroid oriented with the $a$ semiaxis parallel to $\boldsymbol{E}_{0}$ is given by ${ }^{7}$

$$
\phi_{S}=-\phi_{0} \psi_{a}(\xi) \alpha_{a} \quad \text { with }
$$

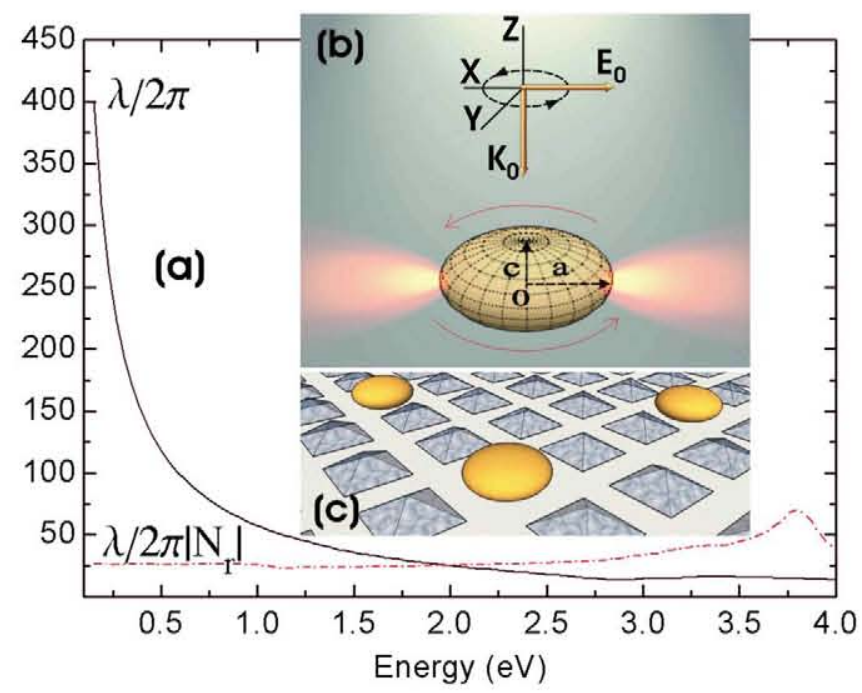

FIG. 1. (Color online) (a) Maximum values of the particle equivalent radius $r_{\mathrm{eq}}$ under EA conditions (1) for Ag particles in GaAs medium. (b) Coordinate system. Light impinges on the particle with its electric field along $x$. The origin $(o)$ is at the particle center. (c) Preferential position for inclusion of MNPs in the plane of the QDs (represented as pyramids). 


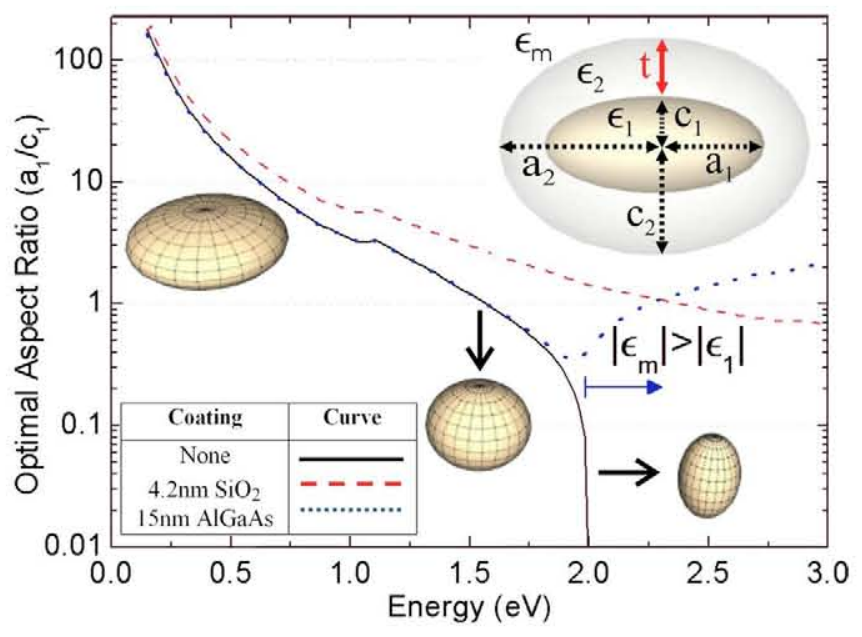

FIG. 2. (Color online) Spheroid aspect ratios that maximize the polarizability for coated and uncoated $\mathrm{Ag}$ MNPs in GaAs medium.

$$
\begin{aligned}
& \psi_{a}(\xi)=\frac{a b c}{2} \int_{\xi}^{\infty} \frac{d q}{\left(a^{2}+q\right) f(q)} \text { and } \\
& f(q)=\sqrt{\left(q+a^{2}\right)\left(q+b^{2}\right)\left(q+c^{2}\right)}, \\
& \alpha_{a}=\frac{\varepsilon_{p}-\varepsilon_{m}}{\varepsilon_{m}+L_{a}\left(\varepsilon_{p}-\varepsilon_{m}\right)},
\end{aligned}
$$

where $\phi_{0}=-E_{0} x$ and $\psi_{a}(\xi)$ is a geometrical factor function of the ellipsoidal coordinate $\xi$. The surfaces $\xi=$ constant are confocal ellipsoids and $\xi=0$ coincides with the particle surface. $\alpha_{a}$ is the polarizability which depends on the difference in dielectric functions between particle $\left(\varepsilon_{p}\right)$ and medium $\left(\varepsilon_{m}\right)$, and on the spheroid aspect ratio $(a / c)$ through the depolarization factor $L_{a}=\psi_{a}(0)$.

The scattered near-field around the MNP is predominantly longitudinal (parallel to its wave vector $\boldsymbol{K}$ ), so energy absorption from this field cannot be described classically such as with far-fields which are transverse (orthogonal to $\boldsymbol{K}) .{ }^{11}$ A longitudinal field is a localized Coulomb field, with no associated Poynting vector, ${ }^{9}$ that transmits electrostatic energy to the medium by means of scalar photons created by the scattered potential operator $\left(\phi_{S}\right)$, as described by quantum electrodynamics. ${ }^{11,12}$ The optimum in absorption by the material in the MNPs near field is thus obtained by finding the particle aspect ratio that maximizes $\left|\phi_{S}\right|$ at each frequency. ${ }^{13}$ For solar frequencies this maximum matches the one of $\left|\alpha_{a}\right|$ and corresponds to the surface plasmon polariton (SPP) resonance. ${ }^{9}$

Figure 2 shows that such SPP aspect ratios decrease with photon energy for Ag MNP in GaAs. However, there is a drawback in inserting metallic particles inside the active region of solar cells since metals may act as recombination centers causing photocurrent degradation. Certain metals might form a Schottky barrier that prevents this to occur. Alternatively, the MNP can be enclosed in a shell of passivating coating with a wider bandgap than the medium. As the refractive index decreases with bandgap, this coating must have a lower refractive index than the host material and will quench the scattered field. Two coatings will be considered: $\mathrm{SiO}_{2}(t=4.2 \mathrm{~nm}$; see inset in Fig. 2), widely used in metaloxide-semiconductor (MOS) technology, and $\mathrm{Al}_{0.4} \mathrm{Ga}_{0.6} \mathrm{As}$

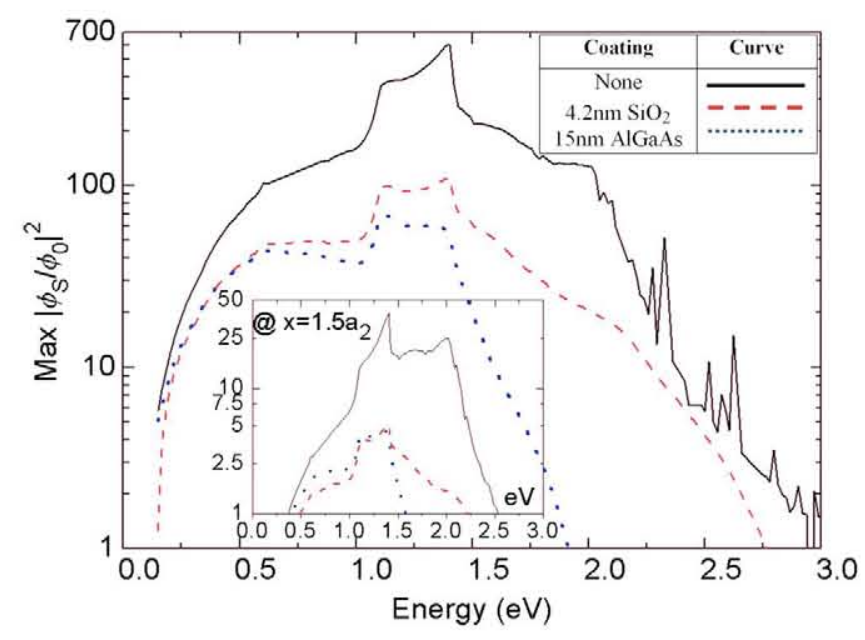

FIG. 3. (Color online) Scattered potential $\left|\phi_{S}\right|^{2}$, in units of $\left|\phi_{0}\right|^{2}$, at the particle surface ( $\xi=0$ or $x=a_{2}$ ) for the SPP shapes in Fig. 2. The inset shows the value of this quantity at $x=1.5 a_{2}$.

$(t=15 \mathrm{~nm})$, a high bandgap III-V alloy that constitutes a good passivating layer for GaAs cells. ${ }^{14}$ The values for the dielectric function of $\mathrm{AlGaAs}$ were taken from http:// refractiveindex.info/. Thicknesses are selected to produce a similar tunnel-leakage current to that acceptable in MOS technology, ${ }^{15}$ considering a metal/insulator/semiconductor rectangular barrier model.

In the electrostatic scattering of double layered ellipsoids, the polarizability becomes ${ }^{\dagger}$

$\alpha_{a}^{\prime}=\frac{\left(\varepsilon_{2}-\varepsilon_{m}\right)\left[\varepsilon_{2}+\left(\varepsilon_{1}-\varepsilon_{2}\right)\left(L_{a}^{(1)}-f L_{a}^{(2)}\right)+f \varepsilon_{2}\left(\varepsilon_{1}-\varepsilon_{2}\right)\right]}{\left[\varepsilon_{2}+\left(\varepsilon_{1}-\varepsilon_{2}\right)\left(L_{a}^{(1)}-f L_{a}^{(2)}\right)\right]\left[\varepsilon_{m}+\left(\varepsilon_{2}-\varepsilon_{m}\right) L_{a}^{(2)}\right]+f L_{a}^{(2)} \varepsilon_{2}\left(\varepsilon_{1}-\varepsilon_{2}\right)}$,

where $\varepsilon_{1}$ and $\varepsilon_{2}$ are the dielectric functions of the core metal and coating, respectively, as sketched in Fig. 2. $L_{a}^{(1)}$ and $L_{a}^{(2)}$ are the corresponding depolarization factors and $f$ $=c_{1} a_{1}^{2} /\left(c_{2} a_{2}^{2}\right)$ is the fraction of the total volume occupied by the inner spheroid. This solution is valid for confocal spheroids, thus the coating thickness is not constant across the particle surface: it is maximal along the minor axis and minimal along the major. Henceforth, the coating thickness $t$ refers to the value along the $c$ axis (see Fig. 2).

For a fixed coating thickness there is an optimal core aspect ratio $\left(a_{1} / c_{1}\right)$ that matches the SPP resonance at each photon energy, maximizing the absolute value of $\mathrm{Eq}$. (3) and $\phi_{S}$. These values are also plotted in Fig. 2 for coated $\mathrm{Ag}$ MNPs in GaAs medium, using the values in Fig. 1(a) for the volume equivalent sphere radius $r_{\mathrm{eq}}$ of the $\mathrm{Ag}$ core. ${ }^{8}$ The coatings considered here do not introduce significant additional restrictions to EA conditions (1) due to their low refractive index (relative to metals) and thickness.

The increment in energy absorption by a system of electrons placed in the near field of MNPs is given ${ }^{13}$ by the quantity $\left|\phi_{S} / \phi_{0}\right|^{2}$, represented in Fig. 3 at the surface of the particles studied in this paper.

This quantity is constant along ellipsoidal surfaces confocal with the particle. Close to oblates it decays roughly with $r^{-6}$ and becomes less than unity after a distance of about twice the particle size $\left(r=2 a_{2}\right)$ in the oblate plane. In the inset of Fig. 3, the value of $\left|\phi_{S} / \phi_{0}\right|^{2}$ is represented at a distance of $1.5 a_{2}$ in this plane. The electric field is even more enhanced in the IR region, reaching $2 \times 10^{5} E_{0}$ at $0.2 \mathrm{eV}$ at the uncoated MNP surface. This is on the same order of the 


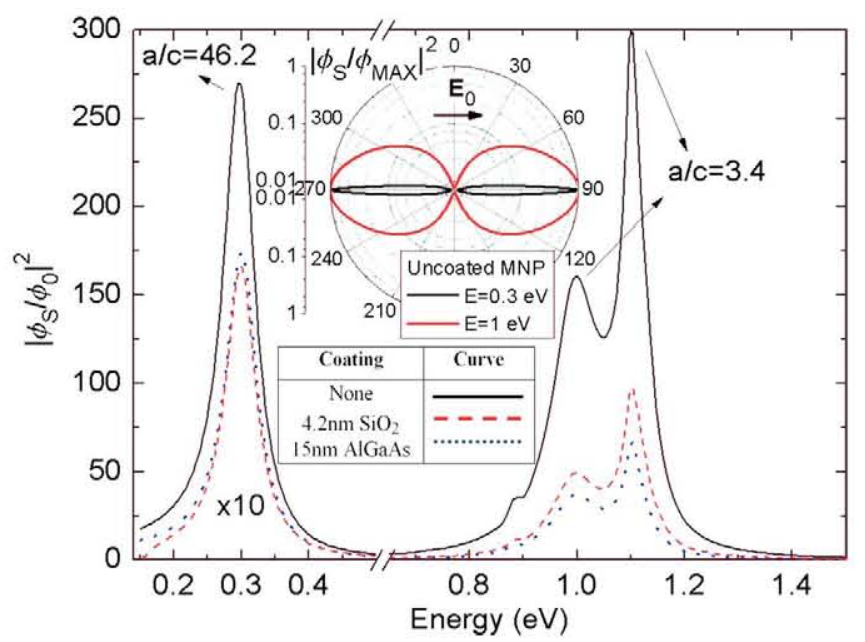

FIG. 4. (Color online) Absorption enhancement for spheroids optimally shaped for 0.3 and $1 \mathrm{eV}$. The curves for the $0.3 \mathrm{eV}$ case are multiplied by 10 for better visualization. The inset is a polar plot of the scattered potential $\phi_{S}$ angular distribution at the uncoated MNP surface in the $x z$ plane, normalized by its maximum at $90^{\circ}$, for the 0.3 and $1 \mathrm{eV}$ shapes.

intrinsic $E$-field between the $p$ and $n$ regions in QD-IBSCs, ${ }^{16}$ therefore the tilt in the QDs band structure caused by the MNPs scattered field is not expected to significantly affect the device performance. This SPP near-field decreases nearly monotonically with photon energy, but evanesces as $r^{-3}$ whereas the far-field evanesces as $r^{-1}$.

The subgap absorption bands in InAs/GaAs QD-IBSC are located at about $1 \mathrm{eV}$ and $0.3 \mathrm{eV}$. Figure 4 shows the spectral absorption enhancement at the surface of MNPs optimally shaped for those energies. The SPP shape corresponding to $1 \mathrm{eV}$ is also optimal for $1.12 \mathrm{eV}$ (see Fig. 2), leading to the broad double peak.

The authors conclude that absorption enhancements in the order of 100 can be expected for QDs close to the uncoated MNPs in the $1 \mathrm{eV}$ absorption band, and around ten with any reasonable coating. For the $0.3 \mathrm{eV}$ band the enhancement, achieved with a set of MNP of different shape, is smaller but still significant in a close vicinity of the particle. Thus, this alternative is most effective if the IBSC mechanism is based on energy transfer through photons absorbed in Valence Band (VB) to IB transitions. ${ }^{17}$ Closely related mechanisms were found to be important in multiple exciton generation materials. ${ }^{18}$
For an MNP shape resonant at a certain photon energy $E$, the field amplification is significant in an energy interval of $E \pm 0.1 \mathrm{eV}$, larger in the $1 \mathrm{eV}$ band.

Within the EA the magnitude of the enhancement is size independent. However, its range is proportional to the MNP size; thus the bigger the MNP the better [under conditions (1) of EA] because enhancement is felt within a larger volume of absorbing medium. MNP sizes in the range of $10-100 \mathrm{~nm}$ fulfill the conditions and should behave adequately.

This work has been supported by the IBPOWER Grant No. 211640 of European Commission, the GENESIS FV Grant No. CSD2006-0004 of the Spanish program CONSOLIDER, and by the NUMANCIA Grant No. S-0505/ENE/ 0310 of Comunidad de Madrid. MJM also acknowledges the Portuguese Government fellowship No. SFRH/BD/21669/ 2005 of FCT-MCTES.

${ }^{1}$ A. Luque and A. Martí, Phys. Rev. Lett. 78, 5014 (1997).

${ }^{2}$ A. Luque, A. Martí, C. Stanley, N. López, L. Cuadra, D. Zhou, and A. Mc-Kee, J. Appl. Phys. 96, 903 (2004).

${ }^{3}$ A. Marti, N. Lopez, E. Antolin, E. Canovas, A. Luque, C. R. Stanley, C. D. Farmer, and P. Diaz, Appl. Phys. Lett. 90, 233510 (2007).

${ }^{4}$ D. Alonso-Alvarez, A. G. Taboada, J. M. Ripalda, B. Alen, Y. Gonzalez, L. Gonzalez, J. M. Garcia, F. Briones, A. Marti, A. Luque, A. M. Sanchez, and S. I. Molina, Appl. Phys. Lett. 93, 123114 (2008).

${ }^{5}$ R. Oshima, A. Takata, and Y. Okada, Appl. Phys. Lett. 93, 083111 (2008).

${ }^{6}$ M. Y. Levy and C. Honsberg, IEEE Trans. Electron Devices 55, 706 (2008).

${ }^{7}$ C. F. Bohren and D. R. Huffman, Absorption and Scattering of Light by Small Particles (Wiley, Weinheim, 2004).

${ }^{8}$ V. V. Somsikov and N. V. Voshchinnikov, Astron. Astrophys. 345, 315 (1999).

${ }^{9}$ L. Novotny and B. Hecht, Principles of Nano-Optics (Cambridge University Press, Cambridge, 2006).

${ }^{10} \mathrm{E}$. Palik, Handbook of Optical Constants of Solids (5 Volume Set) (Academic, San Diego, 1997).

${ }^{11} \mathrm{C}$. Cohen-Tannoudji, J. Dupont-Roc, and G. Grynberg, Photons and Atoms-Introduction to Quantum Electrodynamics (Wiley, New York, 1997).

${ }^{12}$ O. Keller, J. Microsc. 209, 272 (2003).

${ }^{13}$ A. Luque, A. Marti, M. J. Mendes, and I. Tobias, J. Appl. Phys. 104, 113118 (2008).

${ }^{14}$ J. M. Woodall and H. J. Hovel, Appl. Phys. Lett. 21, 379 (1972).

${ }^{15}$ J. Zhang, J. S. Yuan, Y. Ma, and A. S. Oates, Solid-State Electron. 44, $2165(2000)$.

${ }^{16}$ A. Luque, A. Marti, N. Lopez, E. Antolin, E. Canovas, C. Stanley, C. Farmer, and P. Diaz, J. Appl. Phys. 99, 094503 (2006).

${ }^{17}$ A. Luque, A. Martí, and L. Cuadra, IEEE Trans. Electron Devices 50, 447 (2003).

${ }^{18}$ R. D. Schaller, M. Sykora, J. M. Pietryga, and V. I. Klimov, Nano Lett. 6 , 424 (2006). 